\title{
Videodensitometric Methods for Cardiac Output Measurements
}

\author{
Massimo Mischi \\ The Eindhoven Technical University, Electrical Engineering Faculty, Signal Processing Systems Department, \\ Den Dolech 2, P.O. Box 513, 5600 MB Eindhoven, The Netherlands \\ Email:m.mischi@tue.nl

\section{Ton Kalker} \\ The Eindhoven Technical University, Electrical Engineering Faculty, Signal Processing Systems Department, \\ Den Dolech 2, P.O. Box 513, 5600 MB Eindhoven, The Netherlands \\ Email: ton.kalker@ieee.org
}

\section{Erik Korsten}

Catharina Hospital Eindhoven, Department of Anaesthesiology and Intensive Care, P.O. Box 1350, 5602 ZA Eindhoven, The Netherlands

Email: Korsten@chello.nl

Received 1 May 2002 and in revised form 2 October 2002

\begin{abstract}
Cardiac output is often measured by indicator dilution techniques, usually based on dye or cold saline injections. Developments of more stable ultrasound contrast agents (UCA) are leading to new noninvasive indicator dilution methods. However, several problems concerning the interpretation of dilution curves as detected by ultrasound transducers have arisen. This paper presents a method for blood flow measurements based on UCA dilution. Dilution curves are determined by real-time densitometric analysis of the video output of an ultrasound scanner and are automatically fitted by the Local Density Random Walk model. A new fitting algorithm based on multiple linear regression is developed. Calibration, that is, the relation between videodensity and UCA concentration, is modelled by in vitro experimentation. The flow measurement system is validated by in vitro perfusion of SonoVue contrast agent. The results show an accurate dilution curve fit and flow estimation with determination coefficient larger than 0.95 and 0.99 , respectively.
\end{abstract}

Keywords and phrases: contrast agents, local density random walk, ultrasound, videodensitometry.

\section{INTRODUCTION}

The measurement of cardiac blood flow, referred to as cardiac output (CO), is a common practice in the operating room as well as in the intensive care unit. Nowadays the standard techniques for $\mathrm{CO}$ measurements are the thermodilution and the dye-dilution. As these techniques require catheterization, they are considered invasive. The development of sufficiently stable ultrasound contrast agents (UCA) has led to the consideration of their applicability as indicators in dilution techniques $[1,2,3,4]$. UCA are microbubbles (diameter of few $\mu \mathrm{m}$ ) of gas stabilized by a shell of biocompatible material, which are easily detectable by ultrasound analysis [5]. In vitro studies, mainly based on the radio frequency output of the ultrasound scanner, have confirmed that the use of UCA is suitable for flow measurements $[6,7,8,9,10,11]$.

A new method for flow measurements, which can be used for CO estimations, is presented in this study. It is based on the density analysis of the video output of an ultrasound scanner, referred to as videodensitometry. The videodensitometric approach has the advantage of being applicable in every ultrasound scanner since a video output is always available. The same is not true for the radio frequency output, which is available in only a few devices. The mean video density (gray-level) in a selected region of interest (ROI) versus time is recorded to obtain the density-time curve (DTC). Once the DTC is calibrated, that is, when the relation between the video density and the concentration of the contrast has been established, it is referred to as an indicator dilution curve (IDC). The IDC contains all the information for the flow estimation.

Calibration and modeling of the curve are the two crucial issues for a reliable flow measurement. The in vitro calibration shows a range of indicator concentrations where the relation between the video density and the concentration of 
the contrast is linear. Within this range the ultrasound attenuation due to the contrast and the nonlinearity (usually logarithmic compression) introduced by ultrasound scanners can be neglected. The IDC is fitted to a suitable model to determine the parameters of interest. The model and the fitting algorithm have to be robust to the small signal-tonoise ratio (SNR) due to the measurement system and the recirculation of the contrast. The Local Density Random Walk (LDRW) model, which was introduced by Sheppard and Savage in 1951 [12, 13], is adopted to fit the IDC. It gives a physical interpretation of the dilution process [14] and furthermore, although it has never been applied to UCA dilution, it gives the best least squares estimation of the IDC when applied to dye-dilution and thermodilution measurements $[15,16,17,18,19,20]$.

A new fitting algorithm based on multiple linear regression has been developed to fit the IDC by the LDRW model. It allows avoiding the convergence problem of the classical nonlinear fitting algorithms such as Gauss-Newton (GN) and Levember-Marquardt (LM) [21].

A hydrodynamic experimental model is used for the in vitro validation of the method. SonoVue ${ }^{1}$ contrast agent is injected and detected by a transesophageal ultrasound transducer. SonoVue is a new contrast made of microbubbles containing sulfur hexafluoride $\left(\mathrm{SF}_{6}\right)$ and stabilized by phospholipids.

The use of transesophageal echography (TEE) [22] is made in perspective of the in vivo use (in humans) of this technique. This approach improves the SNR since it avoids the noise introduced by ribs and lungs in the classical transthoracic inspection. In addition, since the TEE transducer can be placed almost in touch to the left atrium, it could allow using the in vitro calibration for the in vivo experimentation.

\section{METHODOLOGY}

\subsection{Theory of the LDRW model}

The LDRW model is a monodimensional characterization of the dilution process (see Figure 1). It describes the injection of an indicator into a straight tube where a fluid (carrier) flows with constant velocity $u$. The assumptions are a fast injection and a Brownian motion of the indicator, whose particles interact by pure elastic collisions. Without any loss of generality, we consider the injection time $t_{0}$ and the injection position $x\left(t_{0}\right)$ to be equal to zero. If we focus on the discrete motion of a single particle, its position $X(n T)$ at time $n T$ can be described by the stochastic process given by

$$
X(n T)=\sum_{i=1}^{n} S(i T)
$$

where $S$ is a random variable that represents the distance covered by the particle in the time interval $T$ (single step).

\footnotetext{
${ }^{1}$ SonoVue, trade mark of Bracco Diagnostics (Geneva), information available at http://www.bracco.com/Bracco/Internet/Imaging/Ultrasound/.
}

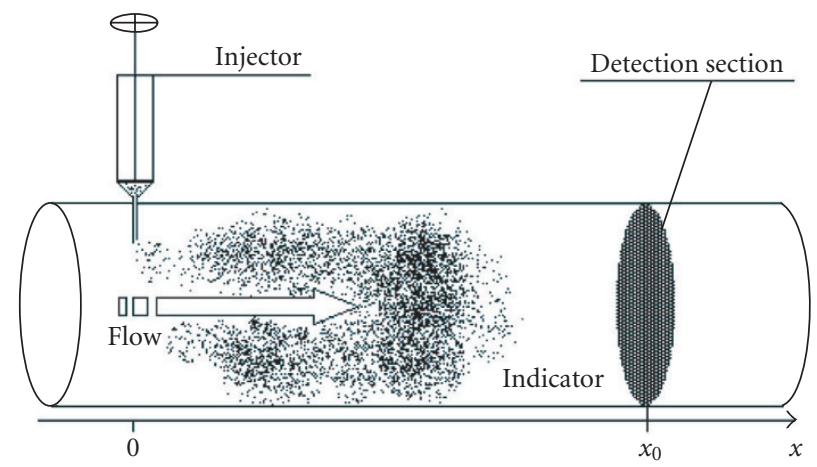

FIGURE 1: LDRW experimental model.

No assumptions are made about the probability density function of the random variable $S$. As a consequence of the Brownian motion hypothesis, each step $S(i T)$ is independent from the previous ones and $X(n T)$ is a Markov process [23]. Therefore, for increasing $n$ (or decreasing $T$ ) we can apply the central limit theorem [24] to the process $X(n T)$. If $\mu$ and $\sigma$ are the mean and the standard deviation of $S$, respectively, then the probability density function of the random variable $X$ at time $n T$ is described by the process $W(x, n T)$ as follows:

$$
W(x, n T)=\frac{e^{-(x-n \mu)^{2} / 2 n \sigma^{2}}}{\sqrt{2 \pi n \sigma^{2}}} .
$$

In terms of continuous time $t=n T$ (with $T$ infinitely small), (2) can be expressed by the Wiener process [25] as

$$
W(x, t)=\frac{e^{-(x-t u)^{2} / 2 t \alpha}}{\sqrt{2 \pi t \alpha}},
$$

where $\alpha=\sigma^{2} / T$ and $u=\mu / T$.

The concentration of the indicator $C(x, t)$ is determined by $(m / A) W(x, t)$, where $m$ is the mass of injected indicator and $A$ is the section of the tube. Thus, $C(x, t)$ is described by a normal distribution that moves along the tube with the same velocity of the carrier (mean equal to $t u$ ) and spreads with a variance that is a linear function of time (variance equal to $\left.t \sigma^{2}\right)$. If we consider $\alpha=2 D$ ( $D$ diffusion coefficient), $C(x, t)$ is the solution of the monodimensional diffusion with drift equation given as

$$
\frac{\partial C(x, t)}{\partial t}=D \frac{\partial^{2} C(x, t)}{\partial x^{2}}-u \frac{\partial C(x, t)}{\partial x}
$$

with the boundary conditions [14]

$$
\begin{aligned}
& C(x, 0)=\frac{m}{A} \delta(x), \\
& \int_{0}^{\infty} C(x, t) d x=\frac{m}{A} .
\end{aligned}
$$

The conditions stated in (5) and (6) express the fast injection hypothesis and the mass conservation law, respectively. Equation (4) represents the link between the statistical and the physical interpretation of the dilution process. In 


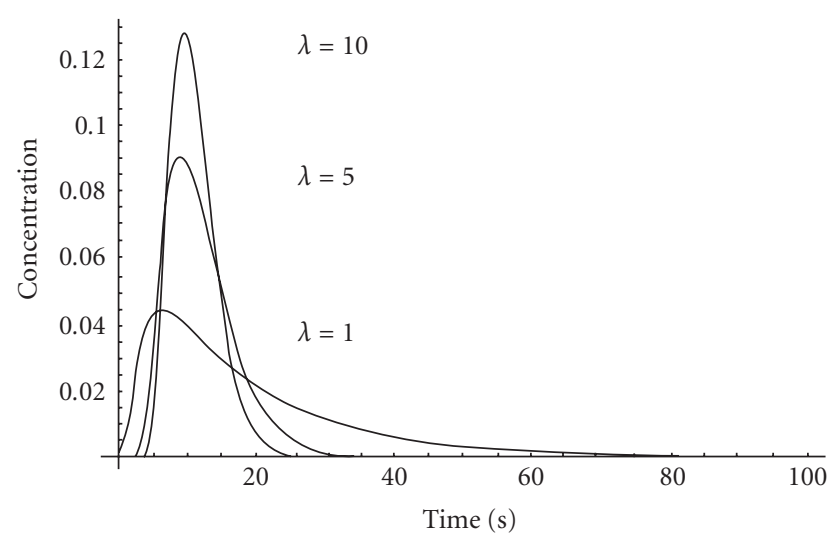

FIgURE 2: Examples of LDRW curves with different $\lambda(\gamma=10$ and $m / q=1)$.

order to obtain a model to describe the IDC, we must focus on a fixed section of the tube (detection section) where the concentration of the indicator is evaluated versus time (see Figure 1). The distance between the injection point and the detection section is determined by $x=x_{0}=u \gamma$. Bogaard et al. $[15,17,18]$ formalized the concentration time curve evaluated at distance $x_{0}$ as

$$
C(t)=\frac{m}{\gamma q} e^{\lambda} \sqrt{\frac{\lambda \gamma}{2 \pi t}} e^{-(\lambda / 2)(t / \gamma+\gamma / t)},
$$

where $q=u A$ is the flow of the carrier and $\lambda=m u^{2} / D=$ $m q^{2} / D A^{2}$ is a parameter related to the skewness of the curve.

For $\lambda>10$ the curve is almost symmetric while for $\lambda<2$ the curve is very skew (see Figure 2). The maximum of $C(t)$ is reached for $t=(\gamma / 2 \lambda)\left(\sqrt{1+4 \lambda^{2}}-1\right)$. Notice that $\max [C(t)]$ is given when $t=\gamma$ only for $\lambda \rightarrow \infty$. It can be explained by the physics of the dilution process. If we consider $L=x_{0}$ as the characteristic length of the LDRW model, we have that $2 \lambda$ equals the Peclet number, which is defined as $u L / D$ and is the hydrodynamic parameter used to quantify the ratio between convection and diffusion in a dilution process $[17,26]$. The limit $\lambda \rightarrow \infty$ can be interpreted as an infinitely small contribution of the diffusion in comparison to the convection. As a consequence, all the particles reach the detection section at the same time $\gamma=x_{0} / u$. Therefore, it is evident that the LDRW model is related to the physical interpretation of the dilution as described by classic hydrodynamics. Some interesting properties of $C(t)$ are

$$
\begin{aligned}
\int_{0}^{\infty} C(t) & =\frac{m}{q} \\
\frac{\int_{0}^{\infty} t C(t) d t}{\int_{0}^{\infty} C(t) d t} & =\gamma\left(1+\frac{1}{\lambda}\right) .
\end{aligned}
$$

The flow $q$ can be directly calculated by ( 8 ) once the injected dose $m$ is known. Equation (9) is the first moment of the LDRW model and it is referred to as the mean transit time (MTT), which is the "mean residence time" of the indicator before the detection distance $x_{0}$ [17]. Once the flow $q$ is known, the volume of fluid between the injection and the detection point is simply given by (MTT) $\cdot q$ (see [13]).

The models that are adopted to fit the IDC are often distinguished between compartmental (cascade of mixing chambers, which includes also the mono-exponential Stewart-Hamilton model) and distributed ones (statistical distributions such as the LDRW model) [16]. However, both of them can be interpreted as impulse response of a mixing system, since the fast injection of the indicator is usually modelled by an impulse. In general, the use of distributed models leads to more precise least square interpolations of the IDC with respect to the compartmental models $[8,15,16,19,20,27]$. Furthermore, among the distributed models, the LDRW, the lognormal, and the $n$-compartmental model, which can be interpreted as a chi-squared distributed model [28], fit the IDC better than the first passage time and the gamma model $[15,18,20]$. As the LDRW model, the first passage model is also based on a random walk of the particles, but it assumes that the detection section is crossed only once $[13,18]$. The reported results and the physical interpretation of the model motivate our choice to adopt the LDRW model to fit the IDC as measured by dilution of UCA.

\subsection{Calibration}

Videodensitometry is based on gray-level measurements. To obtain the IDC out of the videodensitometric analysis, the relation between mean gray-level and real concentration of the contrast must be defined. This relation, referred to as calibration, depends on both the ultrasound intensity that is backscattered by UCA and its conversion into gray levels.

The ultrasound backscatter is defined by the backscatter coefficient $\beta$, which is the scattering cross-section $\left(\mathrm{cm}^{2}\right)$ per unit volume $\left(\mathrm{cm}^{3}\right)$ and per scattering angle ( $\mathrm{sr}$ ). The scattering cross-section of a bubble is the ratio between the power scattered out in all directions and the incident acoustic intensity. If a bubble is approximated by a sphere and its radius changes are described by the Rayleight-Plesset equation $[29,30]$, the scattering cross-section $\sigma$ for a single bubble is a function of the radius $R$ of the sphere and the ultrasound frequency $f$ as given by

$$
\sigma(R, f)=\frac{W(R, f)}{I_{0}(f)}=\frac{4 \pi R^{2}}{\left[\left(f_{r}(R) / f\right)^{2}-1\right]^{2}+\delta_{t}(R, f)},
$$

where $W$ is the scattered power, $I_{0}$ is the incident intensity, and $f_{r}$ is the resonance frequency $[29,31,32,33]$.

The term $\delta_{t}(R, f)$ summarizes all the damping factors. Damping is due to reradiation, viscosity, thermic losses, and-only for shell encapsulated bubbles - internal friction. Since the Rayleight-Plesset equation represents a secondorder system, the scattering cross-section shows a resonance frequency where the system gives the strongest response in terms of scattered power. For $f \gg f_{r}$ it follows that $\sigma(R, f) \simeq$ $4 \pi R^{2}$, which is the physical cross-section, that is, the bubble surface $[32,34]$. The resonance frequency is inversely 
proportional to the radius of the bubbles. Therefore, the total scattering cross-section $\sigma_{\text {tot }}$ depends on the normalized radius distribution $n(R)$ of the bubbles $[31,35]$

$$
\sigma_{\mathrm{tot}}(f)=\int_{R_{\min }}^{R_{\max }} n(R) \sigma(R, f) d R,
$$

where $n(R)$ is a characteristic of the specific contrast. Assuming an isotropic scattering and a low concentration of bubbles, the backscatter coefficient (expressed in $\mathrm{cm}^{-1} \cdot \mathrm{sr}^{-1}$ ) is

$$
\beta(f)=\frac{\rho_{n} \sigma_{\mathrm{tot}}(f)}{4 \pi},
$$

where $\rho_{n}$ is the number of bubbles per unit volume concentration) [31].

Therefore, the backscatter coefficient is a linear function of the UCA concentration $[1,3,8,31,35,36]$ and the backscattered acoustic intensity $I$ that is received by the transducer can be approximated as

$$
I=\frac{V}{r^{2}} \beta(f) I_{0}=\frac{\rho_{n} V \sigma_{\mathrm{tot}}(f)}{4 \pi r^{2}} I_{0},
$$

where $I_{0}$ is the acoustic intensity insonating the contrast, $V$ is the sample volume of contrast, and $r$ is the distance between the contrast sample and the transducer.

Possible experimental solutions for the estimation of $\beta$ are based on the measurement of the ratio between the signal power detected from the contrast and that detected from an acoustic mirror (100\% reflecting layer) when the contrast is absent $[29,36,37]$. This is indeed what is often referred to as integrated backscatter index [36].

The interaction between ultrasound and UCA is not only described by the backscatter coefficient, but also by the increase of the attenuation coefficient, which represents the loss of acoustic pressure in Neper per $\mathrm{cm}$. It is due to the viscous and thermic damping represented by the term $\delta_{t}$ in (10), and by the scattering of acoustic energy into multiple directions. As for the backscatter coefficient, also the attenuation coefficient can be described by a cross-section surface, referred to as extinction cross-section $[29,31,33]$. Since for our application we use low UCA concentrations, we neglect the attenuation effect and we consider the backscattered intensity to be linearly proportional to the UCA concentration, as given in (13).

The relation between mean gray-level and UCA concentration is established once the conversion of the backscattered ultrasound intensity into gray level is defined. The ultrasound transducer performs a linear conversion between ultrasound pressure and electrical voltage. Then, after demodulation, the voltage is quantized into gray-levels by means of a nonlinear relation, which is often implemented as a logarithmic-like compression. In addition, the gamma compensation and the effects of the machine setting (gain and time-gain compensation) should be also taken into account.

Summarizing the linear and logarithmic relations between UCA concentration and gray-levels, we end up with a model to describe the calibration curve. It is defined as

$$
M\left(\rho_{n}\right)=a_{0} \log \left(a_{1} \rho_{n}+a_{2}\right),
$$

where $a_{0}, a_{1}$, and $a_{2}$ are the parameters of the model, and $M\left(\rho_{n}\right)$ is the mean gray-level as a function of the UCA concentration $\rho_{n}$. The term $a_{1} \rho_{n}+a_{2}$ describes the linear relation between backscattered intensity and UCA concentration $\rho_{n}$ (see (13)) and the coefficient $a_{0}$ takes into account the squared relation between voltage and ultrasound intensity as well as the unknown basis of the logarithmic compression.

This model was used to fit the experimental calibration data. The machine setting was kept fixed and a linear gamma was set. The adopted contrast agent was SonoVue. The bubbles contain an innocuous gas $\left(\mathrm{SF}_{6}\right.$, whose molecules are much bigger than $\mathrm{H}_{2} \mathrm{O}$, leading to low diffusiveness) encapsuled in a phospholipidic shell. The bubble size distribution extends from approximately $0.7 \mu \mathrm{m}$ to $10 \mu \mathrm{m}$ with mean value equal to $2.5 \mu \mathrm{m}$ [37]. Studies on the echogenicity ${ }^{2}$ of SonoVue prove that the bigger the bubbles the higher the backscattered power and the lower the resonance frequency $f_{r}$ [32]. As a result, the bubble count is a poor indicator of the efficacy of SonoVue in terms of backscattered power. Much better is the volume evaluation, which is highly related with the amount of bigger bubbles.

SonoVue is delivered in a septum-sealed vial containing $25 \mathrm{mg}$ of lyophilized product in $\mathrm{SF}_{6}$ gas. After the injection of $5 \mathrm{~mL}$ of saline $(0.9 \% \mathrm{NaCl}$ blood isotonic solution) the product is ready for further dilutions. $100 \mathrm{~mL}$ rubber bags were filled with different dilutions of SonoVue in degassed water. The agent was diluted into saline solution. The bags and the TEE ultrasound transducer were plunged in water in order to achieve a good acoustic impedance matching. The B-mode output [38] of the ultrasound scanner was analyzed for all the different dilutions and the mean gray-level measurements were fitted by the model in (14) using an LM algorithm. Since the SonoVue bubbles are stable for only a few minutes [37], each measurement must be executed in a short time (about three minutes). In addition, both the mechanical index (MI, ratio between the peak rarefactional pressure expressed in MPa and the square root of the centre frequency of the ultrasound pulse expressed in $\mathrm{MHz}$ [39]) and the number of frames per seconds (FPS) in the machine setting were set low $(\mathrm{MI}=0.3$ and FPS $=25)$ in order to avoid bubble disruption. The burst carrier frequency was $5 \mathrm{MHz}$ and only fundamental harmonic imaging was used.

The model is tested on two different scanners to verify the machine independency of the model. Figure 3 shows the results as measured with a Sonos 4500 and a Sonos 5500 ultrasound scanners. The determination coefficient $\left(\rho^{2}\right.$, correlation coefficient squared) between the experimental data and the fitted model is 0.99 and 0.98 , respectively. Sometimes, due to the presence of air bubbles, it was difficult to establish the background mean gray-level and therefore the correct

\footnotetext{
${ }^{2}$ Echogenicity is the capability of the contrast to generate echoes when interacting with pressure waves.
} 


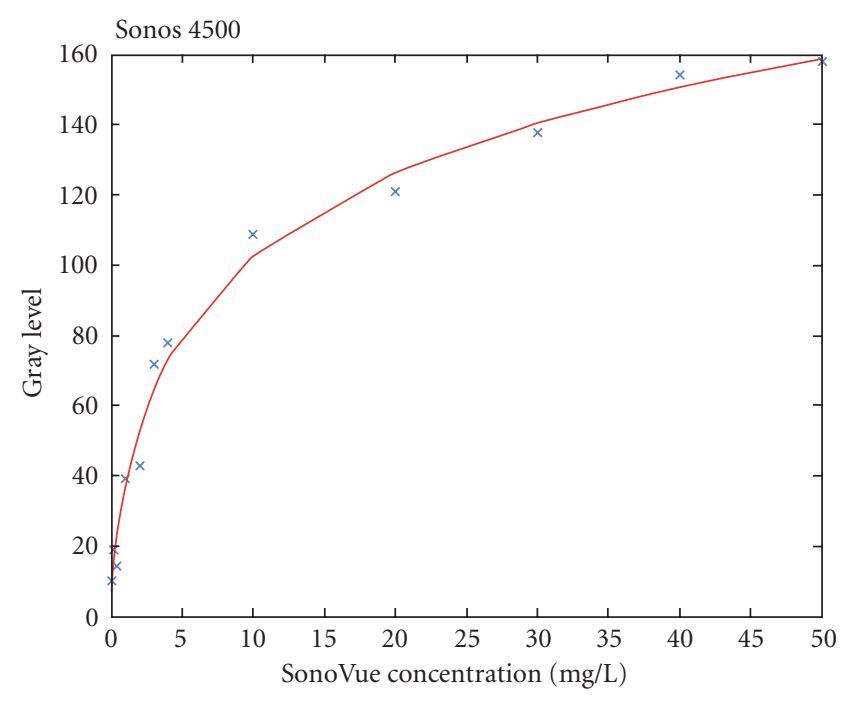

(a)

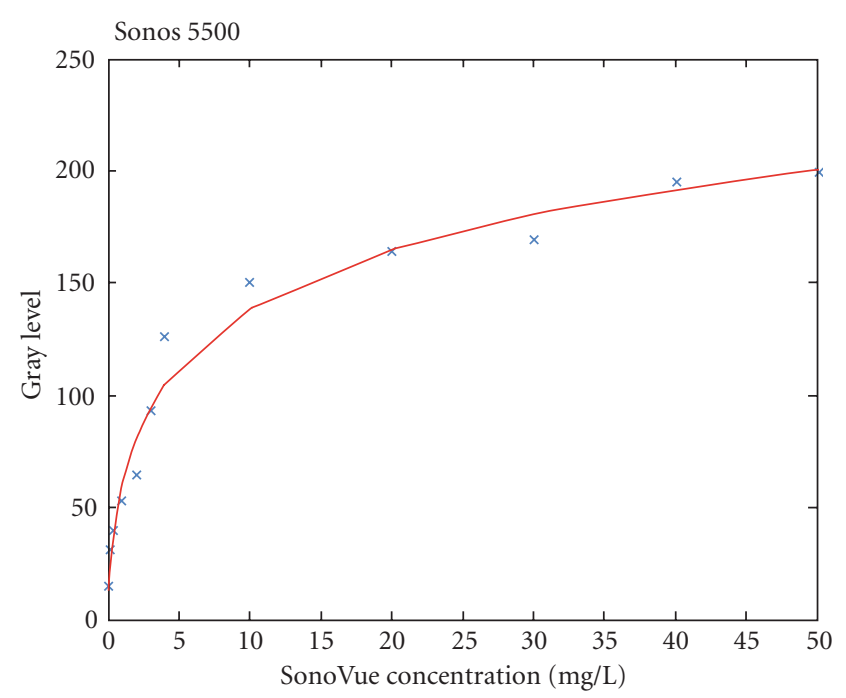

(b)

Figure 3: (a) The calibration fit for the Sonos 4500 scanner. The machine setting was $\mathrm{MI}=0.3, \mathrm{FPS}=25$, burst carrier frequency $=$ $5 \mathrm{MHz}$, gain $=44$, and time gain $=50$. The estimated parameters of the fitted model are $a_{0}=83.1, a_{1}=1.6$, and $a_{2}=1.2$. The determination coefficient is 0.99 . (b) The calibration fit for the Sonos 5500 scanner. The machine setting was $\mathrm{MI}=0.3, \mathrm{FPS}=25$, burst carrier frequency $=5 \mathrm{MHz}$, gain $=44$, and time gain $=50$. The estimated parameters of the fitted model are $a_{0}=90.5, a_{1}=3.2$, and $a_{2}=1.4$. The determination coefficient is 0.98 .

measurements for very low UCA concentrations. Since the logarithmic fitting is very sensitive to the low-concentration data, it is not easy to apply the nonlinear calibration for flow measurements.

Instead, it is interesting to notice that for low concentrations of SonoVue (below $5 \mathrm{mg} \cdot L^{-1}$ ), the relation between UCA concentration and mean gray-level can be approximated by a linear function $M\left(\rho_{n}\right)=a_{0}+a_{1} \rho_{n}$ with $\rho^{2} \geqslant 0.95$. Therefore, we use this simple linear relation for

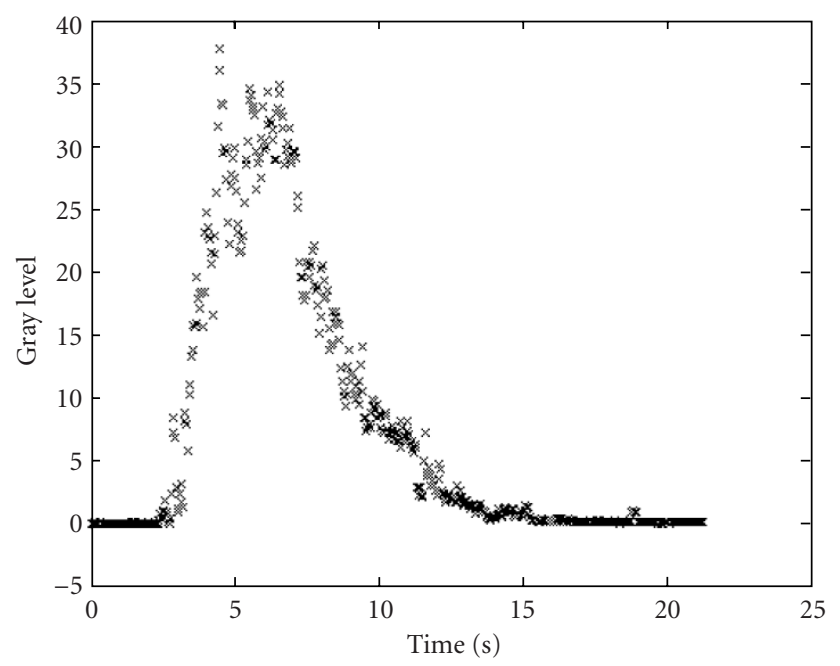

FIgure 4: Example of noisy IDC.

the conversion of the DTC into IDC. As a consequence, in terms if fitting performance, it does not make sense to distinguish between IDC and DTC. In fact, under linear calibration hypothesis, the DTC could be fitted before calibration without any loss of accuracy.

\subsection{Fitting of the LDRW model}

The algorithms that are used for the LDRW model fit are based on either LM or GN nonlinear least square interpolations. The LM and the GN interpolations are recursive algorithms and need an initial estimation of the parameters [21]. Such an estimation is usually performed by the inflection triangle technique or by linear regression to a segment of the ln-transformed IDC (transformed by natural logarithmic function $\ln )[19,40]$. The inflection triangle is constructed by the tangents to the inflection points of the IDC and its shape can be related to the parameters of the LDRW model [18]. The disadvantage of the nonlinear fitting is the convergence problem. In fact the convergence time is strongly dependent on the initial estimation of the parameters. Furthermore, when the IDC (or the DTC) is noisy (see Figure 4), the fitting can converge into local minima. The uncertainty about the injection time $t_{0}$ adds even more complexity.

Therefore, we have developed a new automatic fitting algorithm that is based on multiple linear regression. No assumptions are needed on the input IDC and the injection time. The calibrated density-time signal $C(t)$, which contains the IDC, is processed in two main phases.

In the first phase $C(t)$ is filtered by a low-pass FIR filter (finite impulse response, impulse response defined by $h(t)$ ) to remove the high frequency noise introduced by the measurement system. Then the filtered signal $G(t)=h(t) * C(t)$ is used to determine the position of the IDC within the signal $C(t)$. The time coordinate $t_{\max }$ of the maximum of $G(t)$ is determined, and based on this value the time interval for performing the multiple linear regression is established. This regression time interval is defined for $t \in\left[t_{\text {start }}, t_{\text {end }}\right]$, with $G\left(t_{\text {start }}\right)=0.1 G\left(t_{\max }\right)$ along the rising edge of $\mathrm{G}(t)$ and 
$G\left(t_{\text {end }}\right)=0.3 G\left(t_{\max }\right)$ along the descending edge of $\mathrm{G}(t)$, which is the recirculation appearance time [19]. In perspective of future in vivo applications, the recirculation appearance time defines the time when the contrast reappears into the ROI after a first passage through all the circulatory system.

The initial part of $C(t)$ contains only background noise from the measurement system while the IDC is absent. Furthermore, especially when $\gamma$ is large, the first part of the IDC shows a low SNR (smaller than $-50 \mathrm{~dB}$ ). As a consequence, it is impossible to determine the injection time $t_{0}$ by a simple analysis of either $G(t)$ or $C(t)$. The definition of $t_{\text {start }}$ ensures that $t_{\text {start }}>t_{0}$ and therefore that the regression interval does not include the low-SNR initial part of the IDC.

Before starting the linear fitting, the baseline of $C(t)$ is estimated and subtracted. It is estimated as the mean of $C(t)$ calculated in an early time interval with $t<t_{0}$.

Once the interval $\left[t_{\text {start }}, t_{\text {end }}\right]$ is defined and the baseline is adjusted, in the second phase of the fitting process $C(t)$ and the LDRW model are ln-transformed to obtain a linear model and to apply the multiple linear regression. The resulting linear model is given as

$$
\begin{gathered}
\ln \left(C\left(x_{1}\right)\right)+\frac{1}{2} \ln \left(x_{1}\right)=P_{1}-P_{2} x_{1}-P_{3} x_{2}, \\
x_{1}=t-\hat{t}_{0}, \quad x_{2}=\frac{1}{t-\hat{t}_{0}}, \\
P_{1}=\lambda+\ln \left(\frac{m}{\gamma q}\right)+\frac{1}{2} \ln \left(\frac{\lambda \gamma}{2 \pi}\right), \\
P_{2}=\frac{\lambda}{2 \gamma}, \quad P_{3}=\frac{\lambda \gamma}{2}
\end{gathered}
$$

where $P_{1}, P_{2}$, and $P_{3}$ are the parameters to be optimized, $x_{1}$ and $x_{2}$ are the variables of the linearized model, and $\hat{t}_{0}$ is the estimate of $t_{0}$. The least squares estimation of the parameters $P_{1}, P_{2}$, and $P_{3}$ is solved by (17) [41], which gives the optimum estimation $\underline{P}^{\mathrm{opt}}=\left[\begin{array}{lll}P_{1}^{\mathrm{opt}} & P_{2}^{\mathrm{opt}} & P_{3}^{\mathrm{opt}}\end{array}\right]$. The matrix $[X]$ and the vector $Y$ are defined as shown in (16), where $x_{i 1}$ and $x_{i 2}(i \in$ $[1 \cdots n])$ are the $n$ samples of $x_{1}=t-\hat{t}_{0}$ and $x_{2}=\left(t-\hat{t}_{0}\right)^{-1}$ in the regression interval

$$
\begin{gathered}
\underline{P}^{\mathrm{opt}}=\left([X]^{t}[X]\right)^{-1}[X]^{t} \underline{Y}=\left[\begin{array}{c}
P_{1}^{\mathrm{opt}} \\
P_{2}^{\mathrm{opt}} \\
P_{3}^{\mathrm{opt}}
\end{array}\right], \\
{[X]=\left[\begin{array}{ccc}
1 & x_{11} & x_{12} \\
1 & x_{21} & x_{22} \\
\vdots & \vdots & \vdots \\
1 & x_{n 1} & x_{n 2}
\end{array}\right],} \\
\underline{Y}=\left[\begin{array}{c}
\ln \left(C\left(x_{11}\right)\right)+\frac{1}{2} \ln \left(x_{11}\right) \\
\ln \left(C\left(x_{21}\right)\right)+\frac{1}{2} \ln \left(x_{21}\right) \\
\vdots \\
\ln \left(C\left(x_{n 1}\right)\right)+\frac{1}{2} \ln \left(x_{n 1}\right)
\end{array}\right]
\end{gathered}
$$

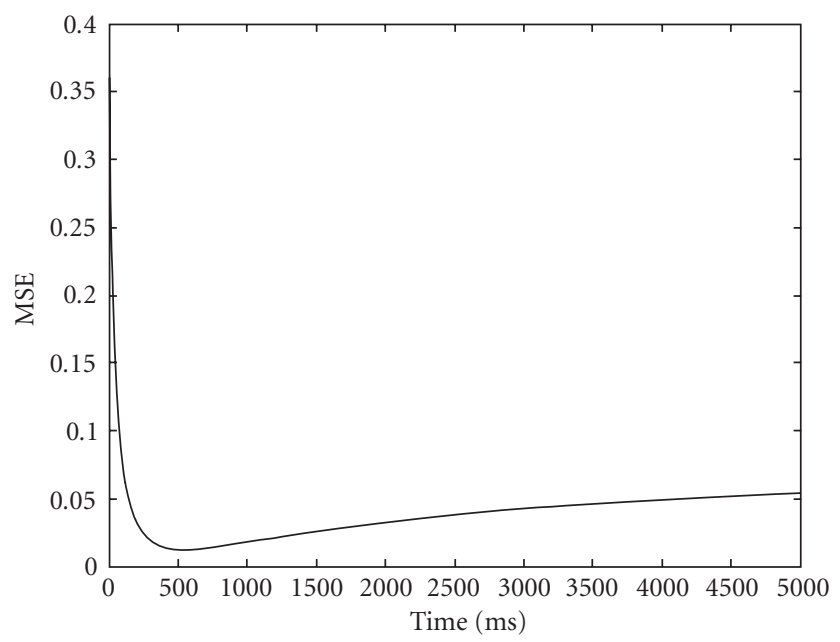

FIGURE 5: MSE of the fitting in the regression interval as function of $t_{\text {start }}-\hat{t}_{0}$.

However, $t_{0}$ is not determined yet. Since by definition $t_{\text {start }}>t_{0}$, the optimum $t_{0}$ can be estimated by applying (16) and (17) recursively for decreasing values of $\hat{t}_{0}$ staring from $\hat{t}_{0}=t_{\text {start }}$ until the minimum square error of the fit is found. This technique is based on the characteristic relation between the mean square error (MSE) of the fit and $\hat{t}_{0}$ $\left(\hat{t}_{0} \leq t_{\text {start }}\right)$. In fact, the relation shows a monotonic behavior and a global minimum for $\hat{t}_{0}=t_{0}$ (see Figure 5). In order to decrease the time-complexity the recursive search is performed by two subsearches with resolution of $40 \mathrm{~ms}$ (as the CCIR system, standard European format [42]) and $1 \mathrm{~ms}$, respectively. Therefore, the final time resolution is $1 \mathrm{~ms}$.

Curves of 2000 samples are fitted in less than $1 \mathrm{~s}$ (Mat$\mathrm{lab}^{3}$ implementation with an AMD $750 \mathrm{MHz}$ processor and 128 MBytes RAM). When $C(t)$ is defined by (7), the curve fit is very accurate.

When $C(t)$ is measured experimentally, it emerges the importance of defining $t_{\text {start }}$ large enough to exclude from the linear fitting the initial part of the IDC. In fact, due to the noise, the limit $t \rightarrow t_{0}$ of $\ln [C(t)]$ does not go to $-\infty$ as expected. Therefore, when the regression interval is close to $t_{0}$, the parameter $P_{3}$, which is a factor of the hyperbole $1 /\left(t-t_{0}\right)$, is not properly estimated. Figure 6 shows $\ln [C(t)]$ in the complete time domain (a) and in the selected regression interval (b). It is evident that the linear regression is not performed on the low-SNR time interval.

The performance of the fitting algorithm is evaluated by adding noise to the theoretical LDRW curve $C_{t}(t)$. The experimental measurements show a modulated white noise, whose amplitude is linearly related to the amplitude of $C(t)$ with $\rho^{2}>0.7$ (see Figure 7). Therefore, artificial white noise $N(t)$ is generated by a random sequence of numbers whose variance (var) is a linear function of $C_{t}^{2}(t)$, that is, $\operatorname{var}[N(t)]=$

\footnotetext{
${ }^{3}$ Matlab 6, trade mark of The Mathworks, information available at http://www.mathworks.com/.
} 


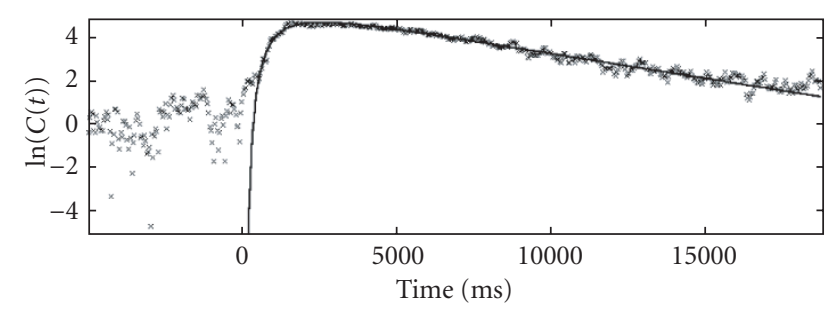

(a)

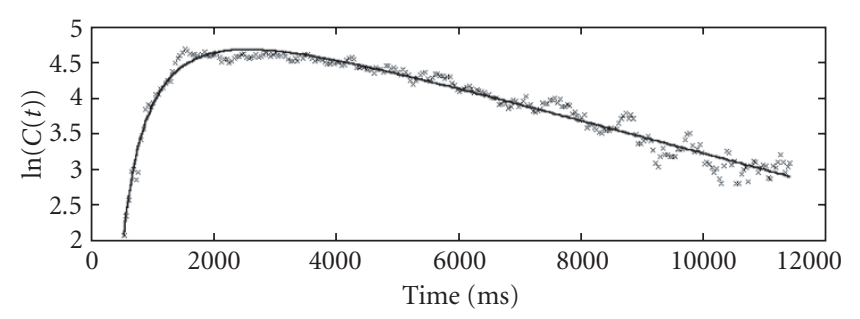

(b)

FIgURE 6: Linear fitting of $\ln [C(t)]$. (a) The fitting in the complete time domain with $t_{0}=0$. Note that $C(t)$ does not go to $-\infty$ when $t \rightarrow 0$ because of the noise. $\operatorname{Re}[\ln [C(t)]]$ is plotted for negative values of $C(t)$. (b) The time interval where the multiple linear regression is performed.

$k C_{t}^{2}(t)$, so that $k$ can be interpreted as $(\mathrm{SNR})^{-1}$. The lowpower background noise is neglected. The fitting of $C_{N}(t)=$ $C_{t}(t)+N(t)$ is performed for $k \in[0 \cdots 1 / 16]$. Lower SNRs have never been met in the experimentation.

The evaluation of the fitting is mainly aimed to study the behavior of the estimation of the area below $C_{t}(t)$ when noise is added. The area below the LDRW model is equal to $\mathrm{m} / \mathrm{q}$ (see (8)), which is indeed the only parameter involved in the flow measurement. Once the vector $\underline{P}^{\text {opt }}$ of (15) and (16) is estimated on the curve $C_{N}(t)$, assuming $M\left(\rho_{n}\right)=\rho_{n}$ (linear calibration with unitary angular coefficient, that is, IDC = DTC), the IDC estimated area is given as

$$
\left[\frac{m}{q}\right]_{e}=\sqrt{\frac{\pi}{P_{2}^{\text {opt }}}} e^{\left(P_{1}^{\text {opt }}-2 \sqrt{P_{2}^{\text {opt }} P_{3}^{\text {opt }}}\right)} .
$$

The estimated area $[\mathrm{m} / \mathrm{q}]_{e}$ of the LDRW fit of $C_{N}(t)$ is compared to the area $m / q$ below $C_{t}(t)$. The results, averaged over 1000 different noise sequences, show a negative bias of $\left([m / q]_{e}-m / q\right)$ that increases with increasing noise (i.e., increasing $k$ ). Notice that bias/ $[\mathrm{m} / q]_{e} \cong \operatorname{bias} /(\mathrm{m} / q)$. No significant differences are appreciated for different $\lambda(\lambda \in$ $[1 \cdots 10])$. Different $\gamma$ and $m / q$ also lead to the same results. Therefore, the results obtained for different $\lambda$ are averaged as shown in Figure 8 and interpolated by linear regression $\left(\rho^{2}>0.9990\right)$. The resulting linear relation between (bias $\left./[\mathrm{m} / \mathrm{q}]_{e}\right)$ and $k$ is

$$
\frac{\text { bias }}{[m / q]_{e}} \cong 0.5255 \cdot k
$$

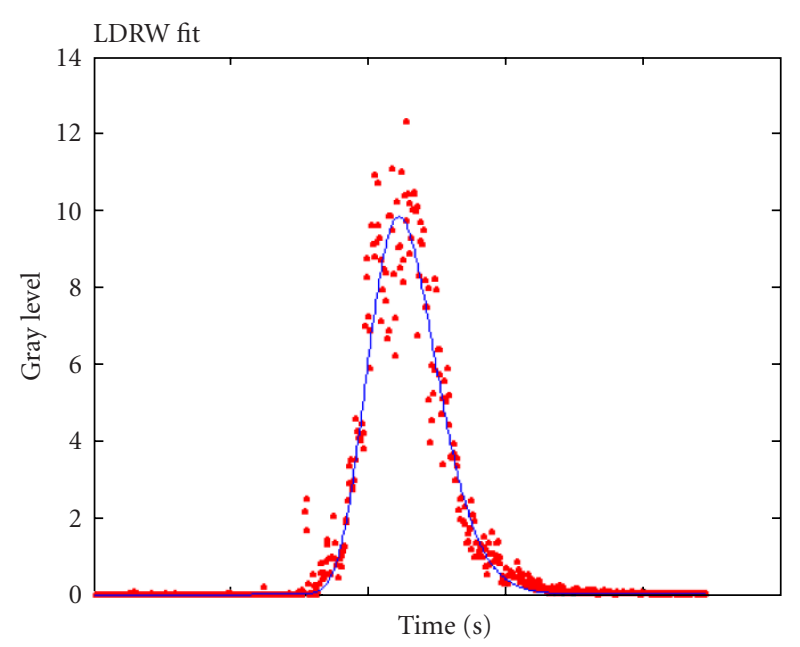

(a)

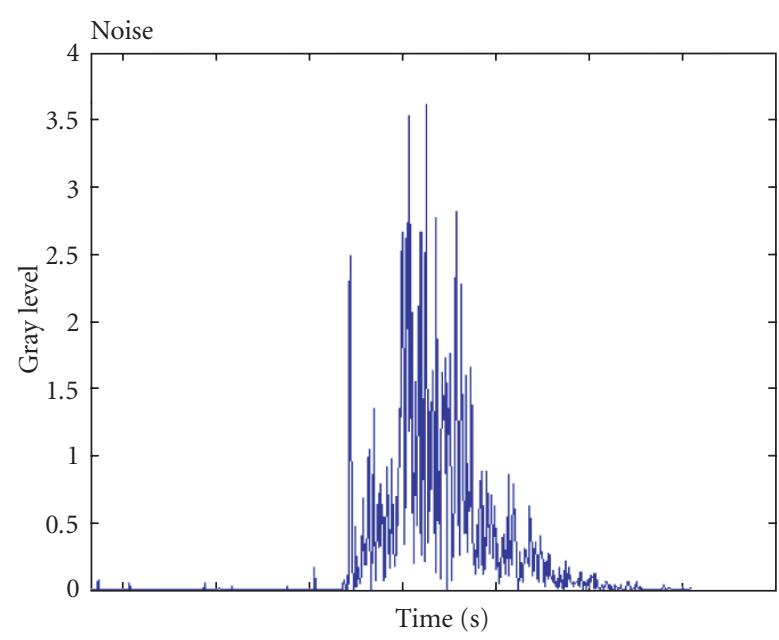

(b)

FIgURE 7: A measured IDC with fitted LDRW model (a) and the absolute value of the difference between the model and the experimental data (b). The noise is clearly correlated to the signal amplitude.

The bias described in (19) can be explained as an effect of the ln-transformation of $C_{N}(t)$ before the linear fitting, which changes the error metrics. In fact, the lntransformation compresses the positive noise more than the negative one. As a consequence the fitted curve is lower than the original $C_{t}(t)$, resulting in a reduced area below the curve (see Figure 9).

Based on (19), a compensation of this effect is implemented in the fitting algorithm. $k$ is determined as the variance of the difference between the LDRW fit and $C(t)$ estimated where the LDRW fit is larger than $95 \%$ of its peak-divided by the squared value of the LDRW-fit peak. When the fitting includes the compensation algorithm, no bias is found in the estimation of the integral of $C(t)$. Figure 9 gives an example of compensation in case of high power noise $(k=1 / 16)$. 


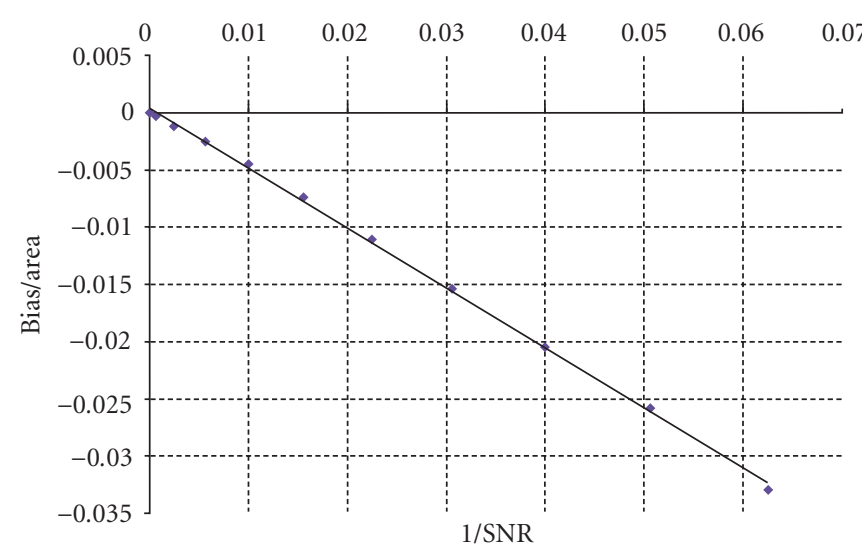

FIgURE 8: The increase of the negative bias (normalized to the estimated area $\left.[\mathrm{m} / \mathrm{q}]_{e}\right)$ as function of $k$.

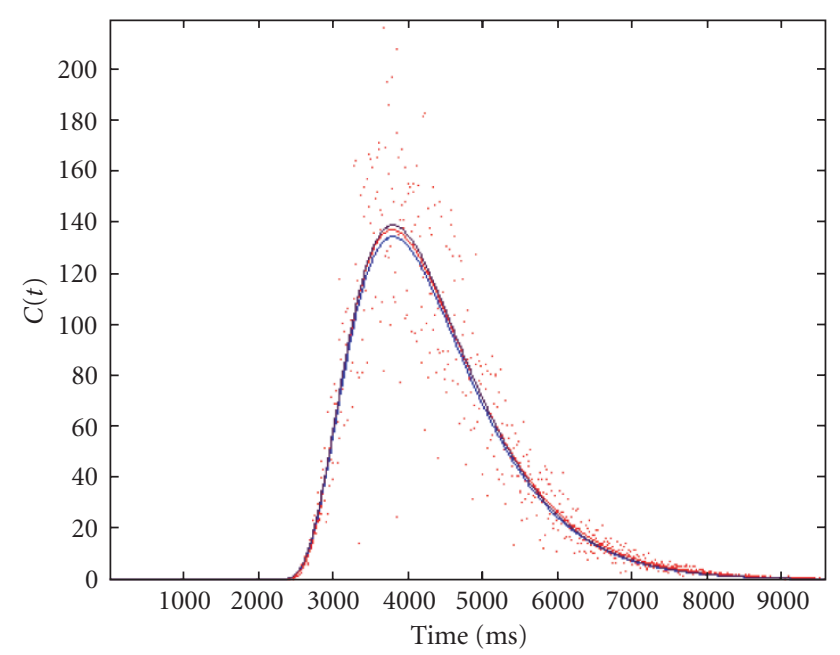

FIgURE 9: Simulation of the fitting. The dots and the upper curve represent the LDRW model $(\lambda=5)$ with and without added noise $(k=1 / 16)$, respectively. The mid and the lowest curves are the LDRW fit of the noise-added curve with and without area compensation.

The variance of $\left([\mathrm{m} / \mathrm{q}]_{e}-\mathrm{m} / \mathrm{q}\right)$ is not affected by the compensation algorithm. It is a linear function of $k(\mathrm{~m} / \mathrm{q})^{2}$. The angular coefficient for $\lambda \in[1 \cdots 10]$ is estimated to be equal to 0.0049 with $\rho^{2}=0.98$. Therefore, in the worst considered case $(k=1 / 16)$ the standard deviation of $\left([m / q]_{e}-m / q\right)$ is equal to $1.75 \%$ of $m / q$. No bias is recognized in the estimation of $\lambda$ and $\gamma$.

\subsection{Experimental in vitro setup}

A hydrodynamic in vitro circuit was built to test the method (see Figure 10). A centrifugal pump and a magnetic flowmeter (clinically used for extracorporeal circulation) generated and measured the flow, respectively. The flow measured by the magnetic flowmeter was the reference to validate the performance of the ultrasound method.

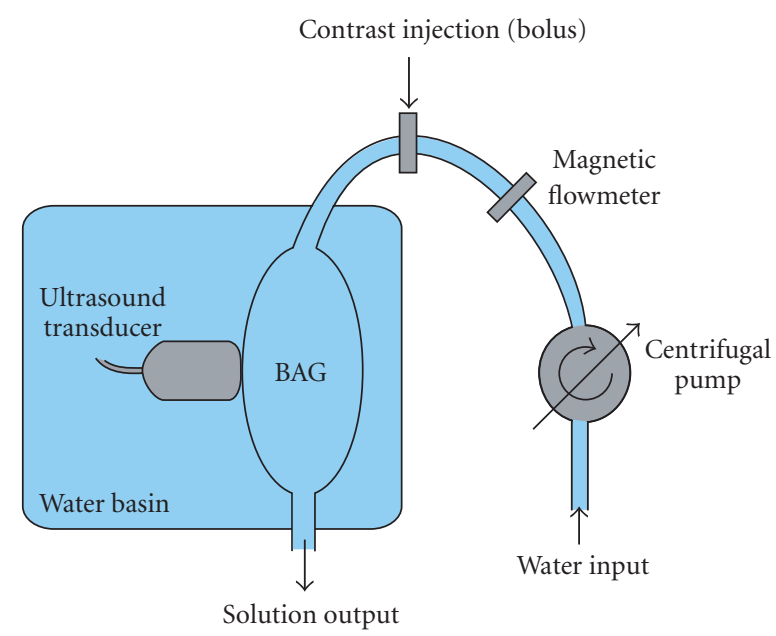

FIGURE 10: Experimental flow-measurement setup.

The contrast injector was positioned after the pump in order to avoid the collapse of the contrast microbubbles due to the turbulence of the pump. The TEE transducer was placed on a plastic bag, considered as a model for a cardiac chamber. The ultrasound transducer and the plastic bag were both plunged in a water-filled basin for the improvement of the acoustic impedance matching.

The adopted ultrasound scanner was a Sonos 4500 (the same machine used for the calibration) equipped with TEE transducer. The centrifugal pump was covered by aluminium foils for magnetic insulation. Such a solution allowed avoiding the interference between the Sonos 4500 scanner and the pump.

A bolus $(10 \mathrm{ml})$ of SonoVue with a concentration of $50 \mathrm{mg} \cdot \mathrm{L}^{-1}$ was injected and detected in B-mode while flowing through the bag. The setting of the ultrasound scanner was the same as adopted for calibration purposes.

A critical part of the setup is the injector. A double injector system was developed in order to avoid air-bubble injections. Several injections of degassed water were used in order to test the system. No air bubbles were detected.

The video output of the ultrasound scanner was grabbed by the $1407 \mathrm{PCI}^{4}$ frame grabber and processed in real time to obtain the DTC, that is, the plot of the mean gray level in the selected ROI versus time. The DTC was then calibrated to obtain the IDC and fitted by the LDRW model. The developed software integrates Labview, ${ }^{5}$ Imaq Vision, ${ }^{6}$ and Matlab implementations.

Since each ultrasound scanner is provided with videorecorder (VCR), it is easy to generate analogical archives

\footnotetext{
${ }^{4} 1407$ PCI, National Instruments, specs available at http://sine.ni.com/ apps/we/nioc.vp?cid=11352\&lang=US.

${ }^{5}$ Labview 6, trade mark of National Instruments, information available at http://sine.ni.com/apps/we/nioc.vp?cid=1385\&lang=US.

${ }^{6}$ Imaq Vision for Labview 6, trade mark of National Instruments, information available at http://sine.ni.com/apps/we/nioc.vp?cid=1301\&lang=US.
} 


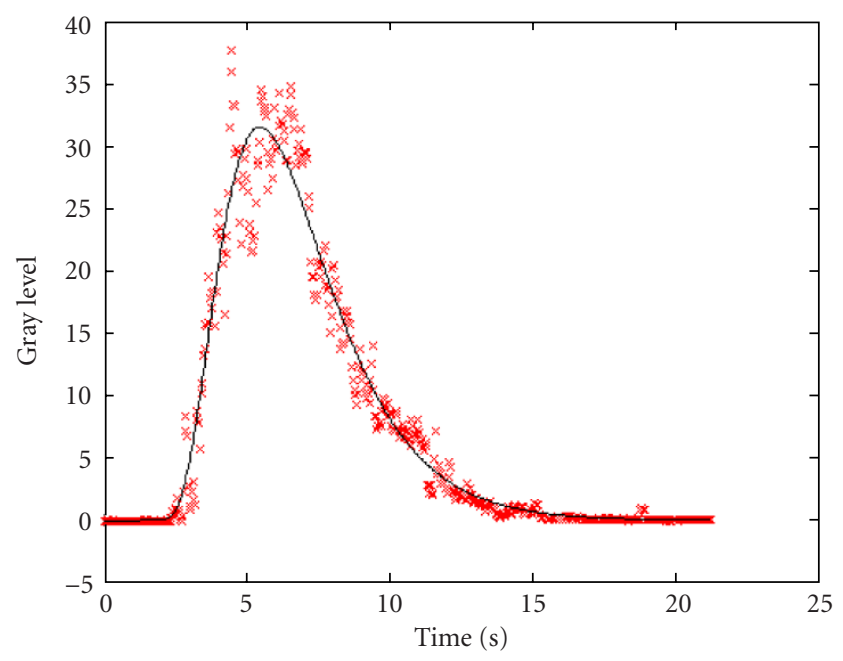

FIgURE 11: LDRW model fit of a DTC, $\rho^{2}=0.97$.

(S-VHS videotapes) and perform further off-line video analysis by playing the videotapes on a VCR.

\section{RESULTS}

The results of the LDRW model fit of the measured DTCs give $\rho^{2}>0.95$. Figure 11 shows the fit of the DTC in Figure 4 .

The MSE is not considered as a parameter to validate the LDRW model since it depends on the noise power. Instead, the MSE is used to compare the developed linear fitting algorithm to the standard LM algorithm. The initial values of the LM fitting are the parameters estimated by linear fitting. The results show that the LM algorithm does not improve the MSE of the linear fit.

The flow estimates are validated by comparison with those measured by a magnetic flowmeter inserted in the experimental hydrodynamic circuit (see Figure 10). Boluses of $10 \mathrm{ml}$ of SonoVue diluted 1:100 (i.e., $50 \mathrm{mg} \cdot \mathrm{L}^{-1}$ ) were injected for the flow measurements. In general, in the perfusion bag a peak concentration of $5 \mathrm{mg} \cdot \mathrm{L}^{-1}$ was hardly reached. Therefore, the linear calibration hypothesis was applicable. The results are given in Figure 12. The determination coefficient between the flow measurements executed by UCA dilution and by magnetic flowmeter is 0.9943 .

\section{DISCUSSION AND CONCLUSIONS}

This paper presents a new flow measurement technique based on UCA dilution. The DTC is obtained by videodensitometric analysis of the video output of an ultrasound scanner. In vitro experimentation was carried out in order to define a relation between the DTC and the IDC. The experimental calibration curve shows a concentration interval where the relation between video density and UCA concentration can be approximated by a linear function. Therefore, the flow measurements are performed within this linear interval.

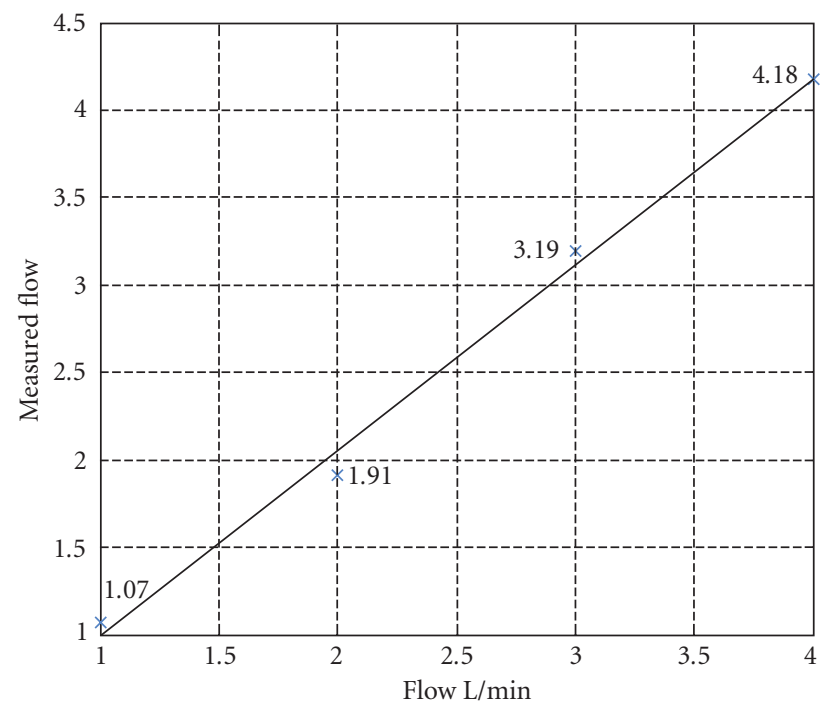

Figure 12: Ultrasound flow measurements ( $Y$-axis) compared to the magnetic flowmeter ones ( $X$-axis). The determination coefficient of the regression line is 0.9943 .

For the first time the LDRW model is adopted to interpolate UCA dilution curves. The chosen model is reported in literature as to give the most accurate IDC fit and a physical interpretation of the dilution process. A new fast linear fitting algorithm has been developed in order to interpolate the IDC by the LDRW model. The results confirm the reliability and robustness of the fitting algorithm as well as the effectiveness of the LDRW model.

The accuracy of the system in the in vitro flow measurements is higher than the accuracy reported for the standard indicator dilution techniques, such as dye- or thermodilution.

Since the system is validated only by in vitro experimentation, the next step will be the in vivo validation. Improvements in the in vitro calibration setup are also planned in order to allow using a nonlinear calibration curve for the flow measurements.

The transesophageal approach allows to place the transducer almost in touch with the left atrium (e.g., in the four chamber view) and therefore to minimize the attenuation. As a consequence, the results of the in vitro calibration could be applied directly to the in vivo measurements.

The advantage of using UCA for in vivo measurements of cardiac output is the low invasiveness of the method, which does not require catheterization. In addition, once the system is able to measure cardiac output, it could be also used for the simultaneous measurements of both ejection faction and distribution volume based on the same indicator dilution principles.

\section{REFERENCES}

[1] C. M. Sehgal and P. H. Arger, "Mathematical modeling of the dilution curves for ultrasonographic contrast agents," Journal of Ultrasound in Medicine, vol. 16, pp. 471-479, 1997. 
[2] D. Rovani, S. E. Nissen, E. Jonathan, et al., "Contrast echo washout curves from left ventricle: application of basic principles of indicator-dilution theory and calculation of ejection fraction," Journal of the American College of Cardiology, vol. 10, pp. 125-134, 1987.

[3] L. Gerfault, E. Helms, V. Bailleau, et al., "Assessing blood flow in isolated pig heart with USCA," in IEEE Ultrasonics Symposium, vol. 2, pp. 1725-1728, Lake Tahoe, Nev, USA, 1999.

[4] T. Gustavsson and M. Beckman-Suurkula, "Videodensitometric measurements in contrast-echocardiography," in IEEE Computers in Cardiology, pp. 709-712, Indianapolis, Ind, USA, September 1996.

[5] H. Becher and P. N. Burns, Handbook of Contrast Echocardiography, Springer, Frankfurt, Germany, 2000.

[6] H. Bleeker, K. Shung, and J. Barnhart, "On the application of ultrasonic contrast agents for blood flowmetry and assessment of cardiac perfusion," Journal of Ultrasound in Medicine, vol. 9, no. 8, pp. 461-471, 1990.

[7] P. A. Heidenreich, J. G. Wiencek, J. G. Zaroff, et al., "In vitro calculation of flow by use of contrast ultrasonography," Journal of the American Society of Echocardiography, vol. 6, pp. 5161, 1993.

[8] X. Chen, K. Q. Schwarz, D. Phillips, S. D. Steinmetz, and R. Schlief, "A mathematical model for the assessment of hemodynamic parameters using quantitative contrast echocardiography," IEEE Trans. Biomedical Engineering, vol. 45, no. 6, pp. 754-765, 1998.

[9] D. Rovai, M. Lombardi, L. Taddei, et al., "Flow quantitation by contrast echocardiography," The International Journal of Cardiac Imaging, vol. 9, pp. 21-27, 1993.

[10] D. Rovai, M. Lombardi, A. Mazzarisi, et al., "Flow quantitation by radio frequency analysis of contrast echocardiography," The International Journal of Cardiac Imaging, vol. 9, pp. 7-19, 1993.

[11] L. J. Bos, J. J. Piek, and J. A. E. Spaan, "Effects of shadowing on the time-intensity curves in contrast echocardiography: a phantom study," Ultrasound in Medicine \& Biology, vol. 22, no. 2, pp. 217-227, 1996.

[12] C. W. Sheppard and L. J. Savage, "The random walk problem in relation to the physiology of circulatory mixing," Physical Review, vol. 83, pp. 489-490, 1951.

[13] C. W. Sheppard, Basic Principles of the Tracer Methods; Introduction to Mathematical Tracer Kinetic, Wiley, New York, NY, USA, 1962.

[14] K. H. Norway, Molecular Dynamics in Biophysics, Paragon Press, Oxford, UK, 1977.

[15] J. M. Bogaard, J. R. C. Jansen, E. A. von Reth, A. Versprille, and M. E. Wise, "Random walk type models for indicator-dilution studies: comparison of a local density random walk and a first passage times distribution," Cardiovascular Research, vol. 20, no. 11, pp. 789-796, 1986.

[16] E. A. Ruth and J. M. Bogaard, "Comparison of a twocompartment model and distributed models for indicator dilution studies," Medical \& Biological Engineering \& Computing, vol. 21, pp. 453-459, 1983.

[17] J. M. Bogaard, S. J. Smith, A. Versatile, M. E. Wise, and F. Homemaker, "Physiological interpretation of skewness of indicator-dilution curves; theoretical considerations and practical application," Basic Research in Cardiology, vol. 79, pp. 479-493, 1984.

[18] M. E. Wise, "Tracer dilution curves in cardiology and random walk and lognormal distributions," Acta Physiol. Pharmacol. Neerl., vol. 14, pp. 175-204, 1966.

[19] R. K. Mallard, Indicator-Dilution Dispersion Models and Cardiac Output Computing Methods, The American Physiological Society, Bethesda, Md, USA, 1997.
[20] E. A. von Reth, Assessment of the indicator-dilution technique in nonstationary flow, Ph.D. thesis, Technical University Eindhoven, Eindhoven, The Netherlands, 1984.

[21] G. A. F. Seber and C. J. Wild, Nonlinear Regression, Wiley, New York, NY, USA, 1989.

[22] J. S. Shanewise, A. T. Cheung, S. Aronson, et al., "ASE/SCA guidelines for performing a comprehensive intraoperative multiplane transesophageal echocardiography examination: recommendations of the American Society of Echocardiography Council for Intraoperative Echocardiography and the Society of Cardiovascular Anesthesiologists Task Force for Certification in Perioperative Transesophageal Echocardiography," Anesth Analg., vol. 89, no. 4, pp. 870-884, 1999.

[23] G. J. M. Uffink, Analysis of dispersion by the random walk method, Ph.D. thesis, Technische Universität Delft, Delft, The Netherlands, 1990.

[24] B. de Finetti, Teoria delle probabilità, vol. 2, Giulio Einaudi, Torino, Italy, 1970.

[25] A. Papoulis, Probability, Random Variables, and Stochastic Processes, McGraw-Hill, Boston, Mass, USA, 1991.

[26] J. W. Delleur, The Handbook of Groundwater Engineering, CRC Press, Boca Raton, Fla, USA, 1999.

[27] J. M. Bogaard, W. A. van Duyl, A. Vesprille, and M. E. Wise, "Influence of random noise on accuracy of the indicator dilution method," Clin. Phys. Physiol. Meas., vol. 6, no. 1, pp. 59-64, 1985.

[28] R. A. F. Linton, N. W. F. Linton, and D. M. Band, "A new method of analysing indicator dilution curves," Cardiovascular Research, vol. 30, pp. 930-938, 1995.

[29] N. de Jong, Acoustic properties of ultrasound contrast agents, Ph.D. thesis, Erasmus University Rotterdam, Rotterdam, Germany, June 1993.

[30] P. J. A. Frinking and N. de Jong, "Modeling of ultrasound contrast agents," in IEEE Ultrasonics Symposium, pp. 16011604, Toronto, Ontario, Canada, 1997.

[31] A. Bouakaz, N. De Jong, L. Gerfault, and C. Cachard, "In vitro standard acoustic parameters of ultrasound contrast agents: definitions and calculations," in IEEE Ultrasonics Symposium, vol. 2, pp. 1445-1448, San Antonio, Tex, USA, 1996.

[32] J. M. Gorce, M. Arditi, and M. Schneider, "Influence of bubble size distribution on the echogenicity of ultrasound contrast agents: a study of SonoVue," Investigative Radiology, vol. 35, no. 11, pp. 661-671, 2000.

[33] N. Sponheim, L. Hoff, A. Waaler, et al., "Albunex-a new ultrasound contrast agent," in IEE conference Acoustic Sensing and Imaging, pp. 103-107, March 1993.

[34] N. de Jong, P. Frinking, F. ten Cate, and P. van der Wouw, "Characteristics of contrast agents and 2D imaging," in IEEE Ultrasonics Symposium, pp. 1449-1458, San Antonio, Tex, USA, 1996.

[35] A. Bouakaz, N. De Jong, and C. Cachard, "Standard properties of ultrasound contrast agents," Ultrasound in Medicine \& Biology, vol. 24, no. 3, pp. 769-772, 1998.

[36] B. Herman, S. Einav, and Z. Vered, "Feasibility of mitral flow assessment by echo-contrast ultrasound, part I: determination of the properties of echo-contrast agents," Ultrasound in Medicine es Biology, vol. 26, no. 5, pp. 785-795, 2000.

[37] M. Shneider, "Characteristics of SonoVue," Echocardiography, vol. 16, no. 7 (pt. 2), pp. 743-746, 1999.

[38] W. R. Hedrick, D. L. Hykes, and D. E. Starchman, Ultrasound Physics and Instrumentation, Mosby, Chicago, Ill, USA, 3rd edition, 1995.

[39] "Information for manifactures seeking marketing and clearance of diagnostic ultrasound systems and transducers," U.S. Department of Health and Human Services, Food and Drug 
Administration, Center for Devices and Radiological Health, November 1997.

[40] J. L. M. Marinus, C. H. Massen, E. A. von Reth, J. M. Bogaard, J. R. C. Jansen, and A. Versprille, "Interpretation of circulatory shunt-dilution curves as bimodal distribution functions," Med. \& Biol. Eng. \& Comp., vol. 22, pp. 326-332, 1984.

[41] W. J. Krzanowski, An Introduction to Statistical Modelling, Edward Arnold, London, UK, 1998.

[42] "Pattern recognition and image processing group, Vienna University of Technology," http://www.prip.tuwien.ac.at/ Research/3DVision/Cameras/tutorial.html.

Massimo Mischi was born in Rome (Italy) in 1973. In 1999, he received his M.S. degree in electronic engineering at La Sapienza University of Rome. In his late thesis he designed and validated a new device for optical-performance measurements of clinical fibre-optic endoscopes. In 2002, he completed a two-year Post-Master program in technological design, information and communication technology, at the Eind-

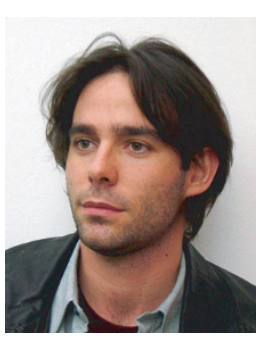
hoven Technical University (the Netherlands). His research was about the application of indicator dilution theory and ultrasound contrast agents for the assessment of cardiac parameters. Now he is a Ph.D. candidate at the Eindhoven Technical University.

Ton Kalker was born in the Netherlands in 1956. He received his M.S. degree in mathematics in 1979 from the University of Leiden, the Netherlands. From 1979 until 1983, while he was a Ph.D. candidate, he worked as a Research Assistant at the University of Leiden. From 1983 until December 1985 he was a Lecturer at the Computer Science Department of the Technical University of Delft. In January 1986 he received

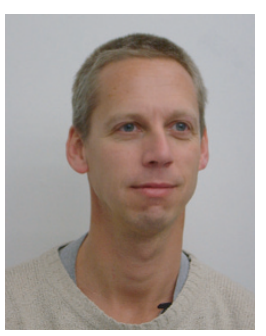
his Ph.D. degree in mathematics. In December 1985 he joined the Philips Research Laboratories, Eindhoven. Until January 1990 he worked in the field of computer aided design. He was specialized in (semi-)automatic tools for system verification. Currently he is a member of the Digital Signal Processing Group of Philips Research. His research interests include wavelets, multirate signal processing, motion estimation, psycho-physics, digital video compression, medical imaging, digital watermarking, and multimedia security. Ton Kalker is a Fellow of the IEEE.

Erik Korsten was born in the Netherlands in 1953. He studied medicine in Groningen and graduated as a medical doctor in 1978 . During his training as an anesthesiologist in Utrecht, he started his Ph.D. study on the measurement of intrathoracic fluid content during open-heart surgery. In November 1984 he received his Ph.D. degree in medicine from the University of Leiden, the Netherlands. From June 1982 until now, he

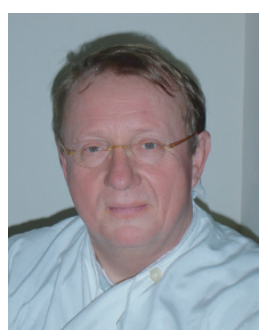
is a staff member of the Department of Anesthesiology, Intensive Care and Pain Treatment in the Catharina Hospital Eindhoven. From 1989 until 1993 he was the Chairman of the professional medical staff of this hospital (a large teaching hospital in the south of the Netherlands). He is also an Intensivist and was head of the Intensive Care during the period 1993 until 2001. During this period he was one of the initiators of a national intensive care database. He was also involved in research projects on data storage and data mining of patient data, as well as the development of artificial intelligence in the intensive care. In 2001 he was appointed as a Professor at the Department of Electrical Engineering, Eindhoven University of Technology, the Netherlands. 